\title{
Necessidade de tomografia computadorizada em pacientes com trauma cranioencefálico de grau leve
}

\author{
The need for computed tomography in patients with minor cranioencephalic trauma
}

Giuliana Ribeiro Lesur ${ }^{1}$, Mariana Baptista Nishida ${ }^{1}$, José Mauro da Silva Rodrigues ${ }^{1}$

\begin{abstract}
RESUMO
Introdução: $\mathrm{O}$ trauma cranioencefálico (TCE), definido como uma lesão no cérebro causado por uma força física externa, é medido pela Escala de Coma de Glasgow (ECG) e pontuado como grave (3-8), moderado (9-13) e leve (14 e 15). O grau TCE está relacionado com a lesão diagnosticada por tomografia computadorizada (TC). No entanto, essa correlação nem sempre ocorre no TCE leve. Objetivo: Identificar o perfil dos pacientes com TCE leve admitidos em um centro de trauma e verificar se o uso rotineiro de TC beneficia esses pacientes. Métodos: Foram analisados 45 laudos de TC. Os dados coletados foram transcritos para o formulário, que continha a avaliação do paciente na ECG como leve, os achados tomográficos e indicações de TC. Resultados: Desses pacientes, 12 foram pontuados com 14 e 33 pacientes com 15. Dentre as indicações de TC, $31(68,9 \%)$ tinham perdido a consciência, $3(6,7 \%)$ pontuaram menos do que 15 até 2 horas da admissão, 1 (2,2\%) tinha suspeita de afundamento de crânio, $7(15,5 \%)$ foram vômitos, $5(11,1 \%)$ tinham idade superior a 65 anos, $14(31,1 \%)$ sofriam de amnésia de eventos antes do trauma e 31 (68,9\%) apresentaram trauma perigoso. Cinco pacientes apresentaram lesões intracranianas e, entre esses, um com possível intervenção neurocirúrgica. Conclusão: Os pacientes com TCE leve, que tenham pelo menos um dos sinais ou sintomas da presença de lesões devem ser submetidos à TC de crânio.

Palavras-chave: traumatismos encefálicos; tomografia computadorizada por raios X; ferimentos e lesões; Escala de Coma de Glasgow.

ABSTRACT

Introduction: Traumatic brain injury (TBI), defined as an injury caused to the brain by an external physical force, is measured by the Glasgow Coma Scale (GCS) and rated as severe (3-8), moderate (9-13) and mild (14 and 15). The TBI level is related to the injury, which is diagnosed by computed tomography (CT); however, this correlation does not always occur in mild TBI. Objective: To identify the profile of patients with mild TBI admitted at a trauma center and to verify if the routine use of CT benefits these patients. Methods: 45 CT reports were analyzed. The data collected was transcribed onto a form, which contained the patient's evaluation using the GCS, for the ones classified as mild trauma patients, CT findings and CT indications. Results: Of these patients, 12 were at level 14 on the GCS and 33 were at level 15. Among the patients that required CT, 31 (68.9\%) had lost consciousness, $3(6.7 \%)$ were below level 15 on the GCS and up to 2 hours after admission, 1 (2.2\%) had suspected skull damage, $7(15.5 \%)$ were vomiting, $5(11.1 \%)$ were older than 65 years, $14(31.1 \%)$ suffered from amnesia of events prior to trauma and 31 (68.9\%) presented dangerous traumas. Five patients presented intracranial injuries and, among these, there was one with possible neurosurgical intervention. Conclusion: Patients with mild TBI, with least one of the signs or symptoms of the presence of injury, should undergo CT of the brain.
\end{abstract}

Keywords: brain injuries; tomography, x-ray computed; wounds and injuries; Glasgow Coma Scale.

${ }^{1}$ Pontifícia Universidade Católica de São Paulo (PUC-SP), Faculdade de Ciências Médicas e da Saúde - Sorocaba (SP), Brasil. Contato: giulesur@hotmail.com

Recebido em 14/06/2016. Aceito para publicação em 20/02/2017. 


\section{INTRODUÇÃO}

O traumatismo cranioencefálico (TCE) é definido como uma lesão causada ao cérebro, por uma força física externa, a qual pode ter como consequência perda ou diminuição da consciência, amnésia ou comprometimento das habilidades cognitivas. O comprometimento funcional pode ser parcial ou total, sendo temporário ou permanente. ${ }^{1,2}$

Entre as principais causas de TCE, podemos citar os acidentes automobilísticos, atropelamentos, acidentes ciclísticos e motociclísticos, agressões físicas, quedas, lesões por arma de fogo, entre outras menos frequentes. ${ }^{3,4}$

O traumatismo craniano é uma das mais comuns contusões no mundo, com uma incidência estimada de 100 a 300 por 100 mil habitantes. As lesões mais leves são definidas como traumatismo contuso da cabeça, após o qual o paciente perdeu a consciência por menos de 15 minutos ou teve uma curta amnésia pós-traumática com duração menor do que 1 hora, ou os dois, mas com uma Escala de Coma de Glasgow (ECG) entre 14 e $15 .{ }^{4}$ Alguns ainda são classificados como trauma leve, se seguidos de sintomas como dores de cabeça, vômitos e, até mesmo, sérios danos faciais, sem necessariamente apresentar amnésia pós-traumática ou perda de consciência. ${ }^{5,6}$

O parâmetro de avaliação da lesão, assim como o seu prognóstico, são feitos principalmente pela ECG. Tal escala, elaborada em 1974 por Graham Teasdale e Bryan J. Jennett, professores de neurologia na University of Glasgow, ${ }^{7}$ atribui pontos ao desempenho do paciente, na abertura dos olhos (4 pontos), nas respostas verbais (5 pontos) e nas respostas motoras (6 pontos), atingindo um total que varia de 3 a 15 pontos, sendo 3 um estado de óbito ou coma e 15 o estado normal de um paciente sem trauma ou sem déficits neurológicos. Os pacientes de 3 a 8 são considerados graves, aqueles com pontuação de 9 a 13, moderados e os demais como leves (14 e 15). ${ }^{1,7,8}$ Antes, pontuação 13 era considerada traumatismo leve, porém, notou-se que os pacientes com ECG 13 geralmente apresentam achados tomográficos e evolução compatível ao TCE moderado. ${ }^{6}$

Para o diagnóstico, a história clínica, o exame físico geral e a avaliação neuropsicológica (se necessário) fornecem informações básicas para estratificação de risco de um paciente ter ou desenvolver lesão neurocirúrgica. Contudo, o principal método de escolha para a avaliação do TCE, juntamente com a ECG e a presença ou não de fratura de crânio, é a tomografia computadorizada (TC) ${ }^{6,9}$

A TC, criada por Hounsfield e Cormack em meados dos anos 1970, ${ }^{10}$ vem diagnosticando TCE no Brasil desde 1977, em São Paulo, no Hospital da Real e Benemérita Sociedade Portuguesa de Beneficência. ${ }^{10}$ As imagens tomográficas demonstram alterações geralmente relacionadas à gravidade da lesão. Fraturas de base de crânio, fraturas craniofaciais, edema cerebral difuso, hematoma subgaleal, hemorragia subaracnoidea, hematoma cerebelar, hematoma epidural e subdural e céfalo-hematoma externo são as principais imagens tomográficas relacionadas ao TCE. ${ }^{5,11}$ Porém, existe uma grande dificuldade em estabelecer uma correlação entre os achados na TC e a gravidade do TCE, mantendo, assim, em todos os trabalhos, a relação de quanto mais grave o TCE, mais numerosos e mais graves os achados na TC. ${ }^{9,11}$

Devido a essa dificuldade de estabelecer um padrão entre achados na TC e a gravidade do TCE, há muitas controvérsias sobre a real necessidade de TC em pacientes avaliados com trauma leve. Embora a TC seja o melhor e mais rápido método para avaliação, e com maior sensibilidade, para alguns, ela não é recomendada como rotina para pacientes com TCE leve. As recomendações variam muito conforme o TCE leve ter alto, médio ou baixo risco para intervenção neurocirúrgica. ${ }^{3,6,8}$

Pacientes com TCE leve de alto risco incluem criança espancada, TCE obstétrico gestante, distúrbio da coagulação e crase sanguínea etc.; fístula liquórica com ou sem débito de líquor; TCE com múltiplos traumatismos; lesões petequiais sugestivas de embolia gordurosa; piora do nível e conteúdo da consciência ou sinais de localização; síndrome de Claud-Bernard-Horner; síndrome de irritação meníngea; distúrbios de funções mentais superiores; déficit de acuidade visual; ferimento por arma branca; e lesão vascular traumática cérvico-craniana. ${ }^{6,8}$

Pacientes com TCE leve de médio risco incluem história de sintomas neurológicos ou envolvimento em acidente grave e/ou com vítimas fatais e/ou história admissional obscura ou não confiável (por exemplo, criança espancada); equimose órbito-palpebral, retroauricular ou lesão significativa de couro cabeludo; quadro clínico sugestivo de intoxicação por álcool ou drogas ilícitas; cefaleia progressiva, vômitos ou convulsão; perda momentânea da consciência; desorientação têmporo-espacial, amnésia retrógrada ou pós-traumática; alterações neuropsicológicas: amnésia pós-traumática; distúrbio da memória visual ou nominativa, distúrbio do comportamento, déficit de atenção ou de concentração; síncope pós-traumatismo (síndrome vaso-vagal); idade inferior a dois anos, exceto se o traumatismo for muito trivial; pacientes com múltiplos traumatismos; suspeita de lesão penetrante ou lesão facial grave; paciente com fratura de crânio, sem lesões intracranianas à tomografia de crânio com anosmia ou anacusia; e paciente de baixo risco que evolui com sintomatologia clínico-neurológica persistente. ${ }^{6,12}$

Pacientes com TCE leve de baixo risco incluem TCE por mecanismo de trauma de pequena intensidade, totalmente assintomáticos; exame físico geral normal, sem alteração neurológica; sinais ou sintomas mínimos; cefaleia discreta e não progressiva; tontura ou vertigem temporária; hematoma subgaleal ou laceração, com raio-X ou TC normal; pacientes com eventual radiografia de crânio que revela alterações, devem ser submetidos à TC de crânio. ${ }^{6,13}$

Alguns estudos mostram positividade para lesões tomográficas em pacientes com sintomas de dor de cabeça e vômitos, idade superior a 60 anos, uso de drogas ou de álcool (intoxicação), déficites de memória de curto prazo ou evidência física de trauma acima das clavículas. ${ }^{3}$

Ainda assim, outros estudos mostram que não há uma correlação estatística entre pacientes com amnésia pós-traumática, dor de cabeça e até mesmo lesão focal encontrada no exame físico com os achados da TC. Entretanto, há fatores como idade avançada, raça branca e fratura basilar, que podem ser correlacionados com alguns achados tomográficos. ${ }^{5}$ 
Baseado nas controvérsias, avaliar os pacientes com TCE leve, os quais têm riscos particulares (alto, médio e baixo risco) de apresentarem alguma alteração na TC, e buscar definir critérios capazes de discriminar a necessidade da realização dessa é o motivo deste trabalho.

\section{OBJETIVOS}

O estudo busca identificar o perfil tomográfico dos pacientes com TCE de grau leve atendidos na Unidade Regional de Emergência do Conjunto Hospitalar de Sorocaba (URE - CHS), São Paulo, observando os fatores de alto, médio e baixo risco para intervenção neurocirúrgica, bem como verificar se a utilização rotineira de TC beneficia esses pacientes.

\section{MÉTODOS}

De forma observacional e retrospectiva, foram avaliados 45 laudos de TC, bem como as fichas de atendimento dos respectivos pacientes, atendidos na URE do CHS, selecionando somente pacientes descritos com TCE leve, cuja ECG fosse 14 ou 15 . As informações foram coletadas em um período de três meses.

Os dados coletados foram descritos em um formulário, elaborado pelos próprios alunos pesquisadores, o qual contém informações sobre a avaliação do paciente na ECG classificados como leve (índices 14 e 15) e o laudo dos achados da TC, além de dados clínicos e pessoais, como: nome, idade, sexo e indicações da TC. Esses dados foram tabulados e avaliados de acordo com a frequência e distribuição percentual.

\section{RESULTADOS}

Inicialmente, a proposta era avaliar 50 fichas de atendimentos e os respectivos laudos das TC. Todavia, foram utilizados no estudo, 45 pacientes, devido à inexistência do exame de imagem e dos laudos, tanto nos prontuários quanto no sistema, de 5 pacientes. Assim, mediante a não informação de existência de lesões intra ou extracranianas, decidiu-se subtrair esses pacientes, utilizando somente aqueles que contemplavam todas as informações exigidas no presente estudo.

Dos 45 casos estudados, quanto ao gênero, $30(66,6 \%)$ eram homens e $15(33,4 \%)$ mulheres; em relação à faixa etária encontramos: menores de 1 ano, 1 (2,2\%); 1 a 14 anos, 9 (20,0\%); 15 a 29 anos, 12 (26,7\%); 30 a 64 anos, $18(40,0 \%)$ e igual ou maior de 65 anos, $5(11,1 \%)$ (Gráfico 1).

Desses pacientes, $12(26,7 \%)$ tinham EGC 14 e $33(73,3 \%)$, ECG 15.

Quanto ao mecanismo de trauma nos quais os pacientes estiveram envolvidos, encontramos: $3(6,7 \%)$ atropelamentos, $5(11,1 \%)$ eventos envolvendo automóveis, $15(33,3 \%)$ eventos envolvendo motocicletas, $19(42,2 \%)$ quedas e $3(6,7 \%)$ vítimas de agressão (Gráfico 2).

Também observamos a quantidade de pessoas envolvidas em cada mecanismo do trauma para cada TCE leve, ou seja, para ECG de 14 e 15 (Gráfico 3).

As indicações de TC muitas vezes foram mais de uma para cada paciente, sendo observado um total de 155 sinais e sintomas para 45 pacientes. Nas indicações tivemos, portanto: $31(68,9 \%)$ perdas de consciência, $3(6,7 \%)$ ECG menor do que 15 até 2 horas da admissão, $1(2,2 \%)$ suspeita de afundamento de crânio, 7 (15,5\%) vômitos, 5 (11,1\%) idade superior a 65 anos, $14(31,1 \%)$ amnésias para fatos anteriores ao trauma e $31(68,9 \%)$ mecanismos de trauma perigoso. Não foram encontrados sinais de fratura de base de crânio (Gráfico 4).

De todos os 45 laudos avaliados, $14(31,1 \%)$ apresentaram sinais de lesão extracraniana, $5(11,1 \%)$ apresentaram

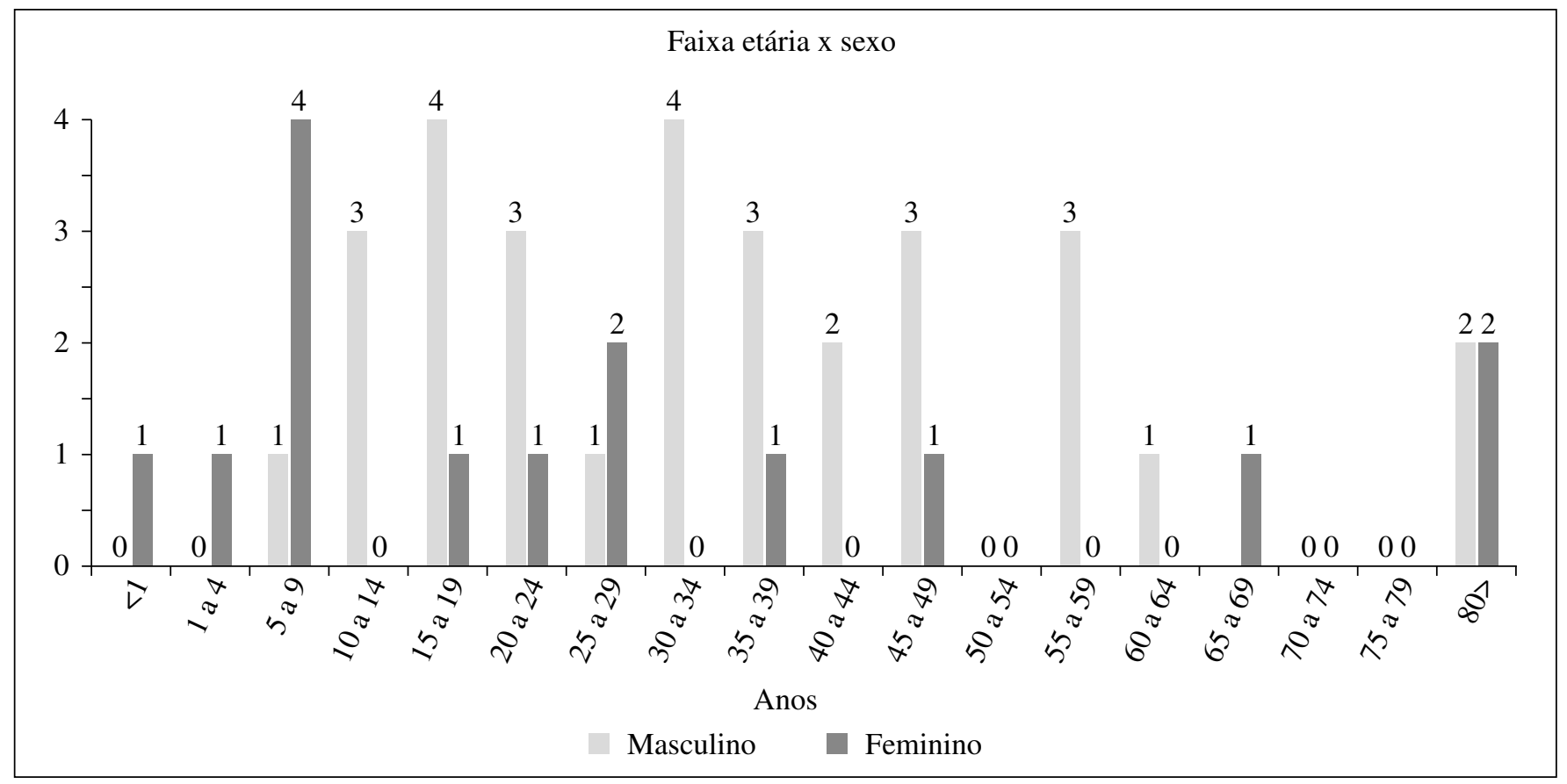

Gráfico 1. Relação faixa etária, de acordo com divisão do DataSUS, e o sexo. 
lesão sugestiva intracraniana e o restante, 26 (57,8\%), não apresentou nenhuma alteração.

Além disso, a avaliação dos laudos permitiu observar que, dos 12 pacientes com ECG 14,1 (8,3\%) apresentou lesão intracraniana; e dos 33 pacientes com ECG 15, encontramos $4(12,1 \%)$ com lesão intracraniana.

Desses 5 pacientes com lesões intracranianas, as indicações de TC mais prevalentes foram: perda de consciência, em 4 (80\%), e mecanismo de trauma perigoso em 3 (60\%), uma vez que mais de uma indicação pode estar presente em um mesmo paciente. (Gráfico 5)

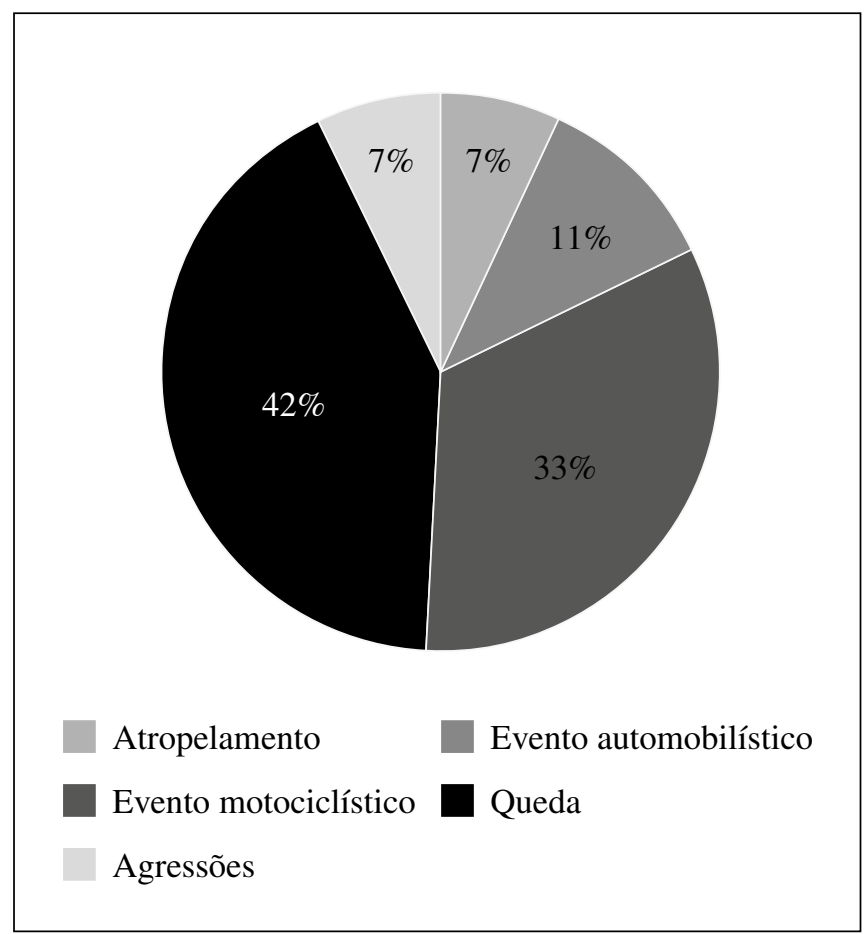

Gráfico 2. Mecanismos do trauma.

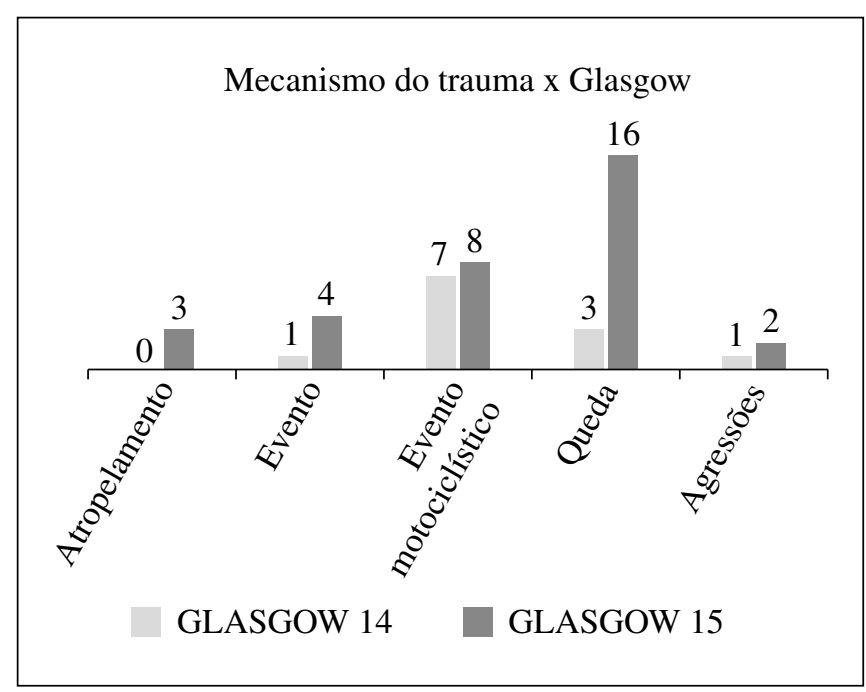

Gráfico 3. Quantidade de pessoas em cada mecanismo de trauma e o tipo de trauma cranioencefálico leve.
Com relação às lesões intracranianas laudadas (5), 2 (40\%) apresentaram aumento de partes moles (edema) e 3 (60\%) com conteúdo hemorrágico, sendo desses, apenas 1 (20\%) com risco hemorrágico e, consequentemente, possível necessidade de intervenção neurocirúrgica. Tal paciente com possibilidade de intervenção neurocirúrgica apresentou TCE leve e ECG 15 na admissão e também apresentou as duas principais indicações de TC de crânio, perda de consciência e mecanismo de trauma perigoso, citadas anteriormente e observadas no Gráfico 5.

\section{CONCLUSÃO}

A TC poderia ser dispensada para os pacientes com ECG 14 ou 15, desde que afastados os critérios de suspeita de lesão que, pela abrangência, estendem as indicações para praticamente todos os casos de trauma.

Avaliando os laudos de todos os pacientes com trauma de crânio leve submetidos à TC e verificando a ocorrência de lesões intracranianas, até mesmo lesões potencialmente cirúrgicas, obtivemos números que justificam, em nossa opinião, a realização do exame.

Assim, acreditamos que os pacientes com trauma de crânio leve, que apresentem ao menos um dos sinais ou sintomas de presença de lesão, devem ser submetidos à TC de crânio.

\section{CONFLITOS DE INTERESSE}

Declaramos que o presente trabalho não apresenta conflito de interesses.

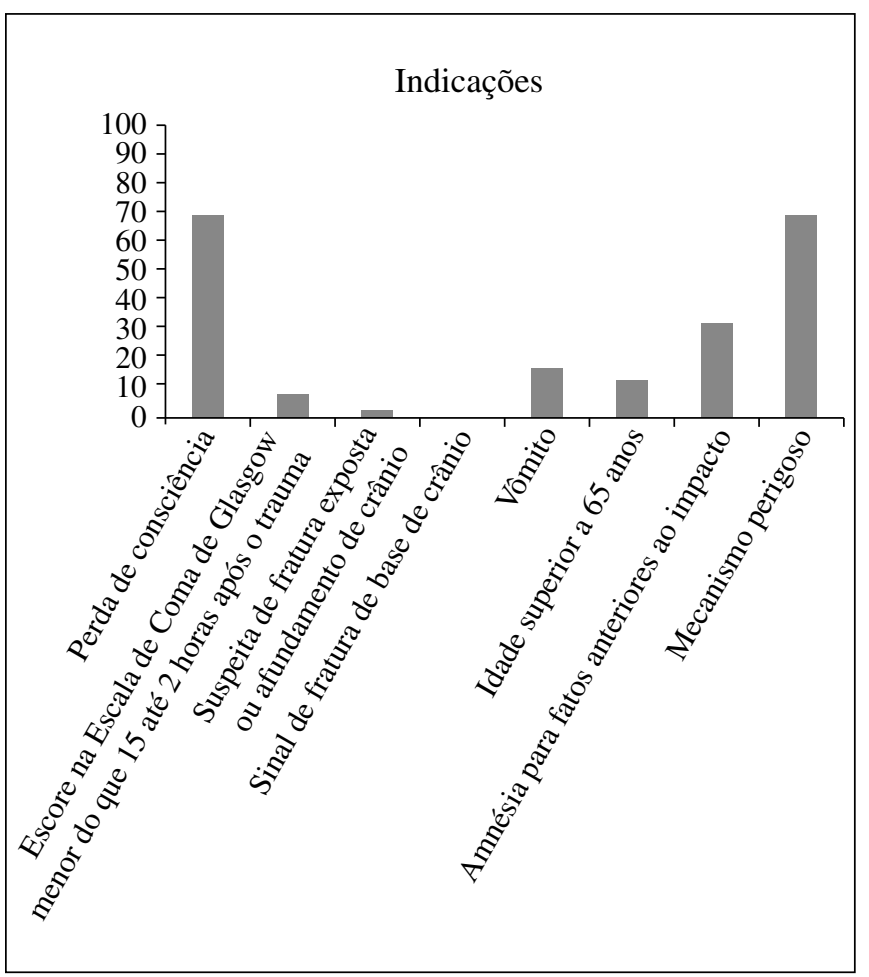

Gráfico 4. Sinais e sintomas que indicaram a realização da tomografia computadorizada. 


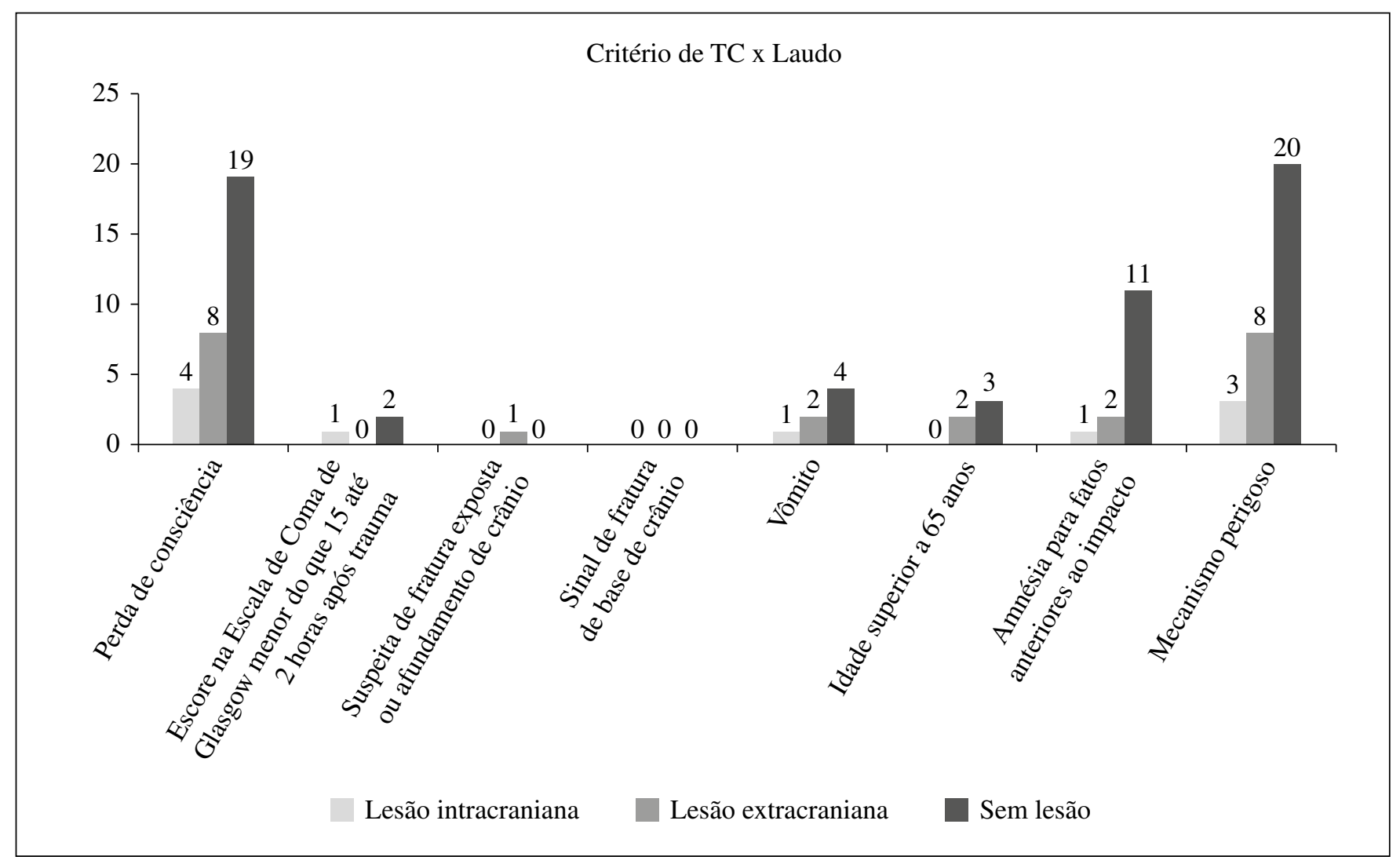

Gráfico 5. Proporção das indicações de tomografia computadorizada em relação ao tipo de lesão encontrada no laudo tomográfico.

\section{REFERÊNCIAS}

1. Rocha CMN. Traumatismo cranioencefálico: correlação entre dados demográficos, escala de Glasgow e tomografia computadorizada de crânio com a mortalidade em curto prazo na cidade de Maceió, Alagoas [tese]. São Paulo: Universidade de São Paulo; 2006.

2. Leite CC, Amaro Jr. E, Lucato LT. Neurorradiologia: diagnóstico por imagem das alterações cefálicas. Rio de Janeiro: Guanabara Koogan; 2008. p.182-214.

3. Haydel MJ, Preston CA, Mills TJ, Luber S, Blaudeau, E, DeBlieux PMC. Indications for computed tomography in patients with minor head injury. $\mathrm{N}$ Engl $\mathrm{J}$ Med. 2000;343:100-5.

4. Smits M, Dippel DWJ, de Haan GG, Dekker HM, Vos PE, Kool DR, et al. External validation of the Canadian CT Head Rule and the New Orleans Criteria for CT scanning in patients with minor head injury. JAMA. 2005;294(12):1519-25.

5. Jeret JS, Mandell M, Anziska B, Lipitz M, Vilceus AP, Ware JA, et al. Clinical predictors of abnormality disclosed by computed tomography after mild head trauma. Neurosurgery. 1993;32(1):9-15.

6. Andrade AF, Marino Jr. R, Miura FK, Carvalhaes CC, Tarico MA, Lázaro RS, et al. Diretrizes de diagnóstico e conduta no paciente com traumatismo cranien- cefálico leve. São Paulo: Sociedade Brasileira de Neurocirurgia; 2001.

7. Teasdale G, Jennett B. Assessment of coma and impaired consciousness: a practical scale. Lancet. 1974;2:81-4.

8 Fundação Hospitalar do Estado de Minas Gerais. Protocolo Clínico de Atendimento ao Paciente Vítima de Traumatismo Cranioencefálico Leve [Internet]. São Paulo: FHEMIG; 2013 [acesso em 25 ago. 2015]. Disponível em: http://formsus.datasus.gov.br/ novoimgarq/15106/2268662_109700.pdf

9. Morgado FL, Rossi LA. Correlação entre a escala de coma de Glasgow e os achados de imagem de tomografia computadorizada em pacientes vítimas de traumatismo cranioencefálico. Radiol Bras. 2011;44:35-41.

10. Carvalho ACP. A história da tomografia computadorizada. Rev Imagem. 2007;29(2):61-6.

11. Miller EC, Holmes JF, Derlet RW. Utilizing clinical factors to reduce head CT scan ordering for minor head trauma patients. J Emerg Med. 1997;15:453-7.

12. Halley MK, Silva PD, Foley J, Rodarte A. Loss of consciousness: when to perform computed tomography? Pediatr Crit Care Med. 2004;5:230-3.

13. Melo JRT, Silva RA, Moreira Jr. ED. Características dos pacientes com trauma cranioencefálico na cidade do Salvador, Bahia. Arq Neuropsiquiatr. 2004;62(3A):711-5. 